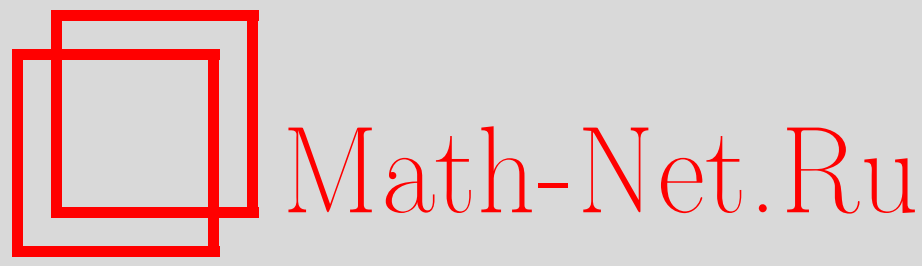

Ю. В. Брежнев, Трансцендентные формулы следов для конечнозонных потенциалов, ТМФ, 2010, том 164, номер 1, 108-118

DOI: https://doi.org/10.4213/tmf6527

Использование Общероссийского математического портала Math-Net.Ru подразумевает, что вы прочитали и согласны с пользовательским соглашением http://www . mathnet.ru/rus/agreement

Параметры загрузки:

IP : 3.95.254.165

26 апреля 2023 г., 12:57:43

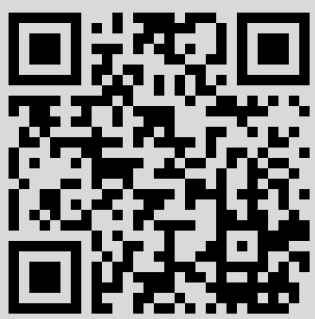




\title{
ФИЗИКА
}

Том 164, № 1

июль, 2010

2010 г.

\author{
Ю. В. Брежнев*
}

\section{ТРАНСЦЕНДЕНТНЫЕ ФОРМУЛЫ СЛЕДОВ ДЛЯ КОНЕЧНОЗОННЫХ ПОТЕНЦИАЛОВ}

Показано, что в теории конечнозонного интегрирования спектральных уравнений имеют место формулы, отличные от классических аналогов рациональных формул следов для алгебро-геометрических потенциалов. Новые формулы содержат трансцендентные модулярные функции и гипергеометрические ряды. Как следствие, они приводят к трансцендентным соотношениям между тета-функциями.

Ключевые слова: спектральная задача, конечнозонный потенциал, формулы следов, модулярная функция.

\section{1. ВВЕДЕНИЕ}

В теории интегрирования спектральных задач известны так называемые тождества и формулы следов. Терминология восходит к работам Крейна, Гельфанда и Дикого 50-х годов по спектральной теории обыкновенного дифференциального уравнения вида $\psi_{x x}-u(x) \psi=\lambda \psi$. В 1975 г. в связи с возникновением методов конечнозонного интегрирования (в спектральной терминологии) В. Матвеев установил новую и важную трактовку этих формул. Упомянутые соотношения, включая найденные $t$-изоспектральные разновидности, представляют собой дифференциальные тождества для точно решаемых потенциалов $u(x)$. В частности, одна из серии таких формул, а именно знаменитая формула

$$
u(x)=2\left\{\gamma_{1}(x)+\cdots+\gamma_{g}(x)\right\}+\text { const }
$$

позволяет восстановить потенциал по величинам $\gamma_{k}(x)$, которые удовлетворяют интегрируемым обыкновенным дифференциальным уравнениям - уравнениям Дубровина. Все оставшиеся тождества следов являются следствием этой “главной" формулы. В настоящей работе мы будем смотреть на эти соотношения, а точнее на их аналоги для спектральных уравнений высших порядков, именно с этой точки зрения: как на некоторые формулы, по которым точно решаемый потенциал выражается через величины $\gamma_{k}$.

\footnotetext{
*Томский государственный университет, Томск, Россия. E-mail: brezhnev@mail.ru
} 
Как известно, потенциал $u(x)$ определяет конечномерную гамильтонову систему, интегрируемую по Лиувиллю, где $x$ выступает как время. Переход к каноническим переменным $(p, q)$ осуществляется путем комбинирования потенциала и его производных: $p=p\left(u, u_{x}, \ldots\right), q=q\left(u, u_{x}, \ldots\right)$ [1]. Далее, переход к переменным разделения - это переход к величинам $\gamma_{k}$ и $\mu_{k}$, связанным алгебраическим соотношением $W\left(\gamma_{k}, \mu_{k}\right)=0$, которое называется уравнением спектральной кривой. Формулы обратного перехода $p=p\left(\gamma_{k}, \mu_{k}\right), q=q\left(\gamma_{k}, \mu_{k}\right)$ и, следовательно, формула вида $u=u(p, q)$ в теории гамильтоновых систем хорошо известны [2], однако наглядный смысл формулы (1) как дифференциального аналога следа оператора имеется только для уравнения Шредингера. "Следовая" трактовка всех других алгебро-геометрических (конечнозонных) операторов не имеет места. Это является проявлением того факта, что в общей теории разделения переменных должны участвовать обе координаты $\gamma$ и $\mu$ спектральной кривой, так как абелевы функции выражаются, вообще говоря, через обе эти переменные. Уравнения второго порядка доставляют лишь специфический и частный случай, когда $u=u(\gamma)$. Для более сложных спектральных задач имеются изолированные примеры [3], а общие схемы построения главной формулы следов

$$
u=R\left(\gamma_{k}, \mu_{k}\right)
$$

с рациональной функцией $R$, насколько нам известно, отсутствуют.

Предметом настоящей статьи является, однако, не этот вопрос, а то наблюдение, что, помимо представления потенциалов как абелевых функций в виде (2), возможны и другие представления. Они даются не рациональными, а трансцендентными, но тоже однозначнъми функциями от симметрических комбинаций координат $\left(\gamma_{k}, \mu_{k}\right)$. Пример, на котором мы демонстрируем этот факт, интересен не только сам по себе, но и тем, что он приводит к новым трансцендентным тождествам между $\Theta$-функциями. Напомним, что полиномиальные тождества между $\Theta$-функциями общеизвестны. Фактически они являются униформизирующими представлениями абелевых многообразий [4] и, как следствие, $\Theta$-функциональными представлениями абелевых функций (2).

\section{2. РАЦИОНАЛЬНАЯ ФОРМУЛА СЛЕДОВ}

Рассмотрим спектральную задачу третьего порядка

$$
\Psi^{\prime \prime \prime}+u(x) \Psi^{\prime}-\frac{1}{2} u^{\prime}(x) \Psi=\lambda \Psi
$$

Известно, что если ассоциировать эту задачу с нелинейным интегрируемым уравнением, то ее изоспектральные деформации описываются уравнением Каупа-Купершмидта [5]

$$
u_{t}=u^{(\mathrm{v})}+5 u u^{\prime \prime \prime}+\frac{25}{2} u^{\prime} u^{\prime \prime}+5 u^{2} u_{x}
$$

В дальнейшем нам достаточно будет ограничиться стационарными решениями этого уравнения или, что тоже самое, рассмотреть коммутирующий с (3) операторный 
пучок, линейно зависящий от $\lambda$,

$$
A(\lambda ;[u]) \Psi^{\prime \prime}+B(\lambda ;[u]) \Psi^{\prime}+C(\lambda ;[u]) \Psi=\mu \Psi
$$

2.1. Алгебраическая кривая. Как обычно, совместность уравнения (5) со спектральной задачей, т.е. с задачей (3), дает выражения для $A, B, C$ :

$$
A=-9 \lambda, \quad B=u^{2}+\frac{1}{2} u^{\prime \prime}+c, \quad C=-\frac{1}{3} A^{\prime \prime}+\frac{2}{3} A u-B^{\prime} .
$$

Константа $c$ связана со стационарной динамикой $u=u(x-c t)$, и мы имеем

$$
-9 \lambda \Psi^{\prime \prime}+\left(u^{2}+\frac{1}{2} u^{\prime \prime}+c\right) \Psi^{\prime}-\left(6 u \lambda+2 u u^{\prime \prime}+\frac{1}{2} u^{\prime \prime \prime}\right) \Psi=\mu \Psi .
$$

Величины $\lambda$ и $\mu$ алгебраически зависимы, т.е. лежат на спектральной кривой

$$
\mu^{3}+Q(\lambda ;[u]) \mu+T(\lambda ;[u])=0
$$

Ее уравнение получается исключением $\Psi$ из (3) и (5). В результате получаем

$$
\begin{aligned}
\mu^{3}+\left(27 c \lambda^{2}-\frac{9}{2} K \lambda\right. & \left.+E_{2}\right) \mu+729 \lambda^{5}+ \\
& +E_{1} \lambda^{3}+\frac{27}{2} u K \lambda^{2}+E_{3} \lambda+\frac{1}{8}\left(u^{\prime \prime}+2 u^{2}+2 c\right)^{2} K=0 .
\end{aligned}
$$

Выражения для $E_{k}$ мы приведем позже, а здесь заметим, что в уравнение кривой входят не только интегралы стационарного уравнения (4), но и само это уравнение

$$
K=c u^{\prime}+u^{(\mathrm{v})}+5 u u^{\prime \prime \prime}+\frac{25}{2} u^{\prime} u^{\prime \prime}+5 u^{2} u^{\prime} .
$$

В силу (4) константа $K$ должна обращаться тождественно в нуль. Но этому не противоречит и выражение $u^{\prime \prime}+2 u^{2}+2 c=$ const. Если константа в правой части этого уравнения не равна нулю, то совместность с уравнением $K=0$ дает тривиальный результат $u=$ const. В противном случае имеем кривую в вырожденной ситуации:

$$
\mu^{3}+27 c \lambda^{2} \mu+729 \lambda^{5}-27\left(\frac{3}{4} u^{\prime 2}+u^{3}+3 c u\right) \lambda^{3}=0 .
$$

Эта кривая имеет род $g=1$, соответствующее решение $u=-3 \wp\left(x+3 g_{2} t / 4\right)$ легко вычисляется, и нас оно не будет интересовать. Для невырожденного случая кривая (6) приобретает вид

$$
\mu^{3}+\left(27 c \lambda^{2}+E_{2}\right) \mu+729 \lambda^{5}+81 E_{1} \lambda^{3}+E_{3} \lambda=0
$$

имеет род $g=4$, и задача определения соответствующего потенциала нетривиальна. 
2.2. Рациональная формула следов. Получим сначала обычную формулу следов, т.е. представление потенциала в виде (2). Прежде всего, следует определить величины $\gamma_{k}$. В теории конечнозонных операторов в качестве таких величин выступают полюсы функции $\Psi(x)$, которые не зависят от $\lambda$. Исключая из уравнений (3) и (5) функцию $\Psi$ и ее первую производную, мы получим выражение

$$
\frac{\Psi^{\prime}}{\Psi}=27 \lambda \frac{3 \mu^{2}+Q}{\Pi}-\frac{1}{18 \lambda}\left(u^{\prime \prime}+2 u^{2}+2 c\right) .
$$

Здесь $Q(\lambda):=27 c \lambda^{2}+E_{1}([u])$, а выражение для дифференциального полинома $\Pi=$ $\Pi(\lambda ;[u])$ выглядит следующим образом:

$$
\begin{aligned}
\frac{1}{3^{7}} \Pi(\lambda ;[u])= & \lambda^{4}-\frac{1}{6} u^{\prime} \lambda^{3}+\frac{1}{18}\left(u^{(\mathrm{IV})}+5 u u^{\prime \prime}+4 u^{\prime 2}+2 u^{3}+2 c u\right) \lambda^{2}+ \\
& +\frac{1}{324}\left(u^{\prime \prime \prime}+4 u u^{\prime}\right)\left(u^{\prime \prime}+2 u^{2}+2 c\right) \lambda+\frac{1}{3^{6} 8}\left(u^{\prime \prime}+2 u^{2}+2 c\right)^{3} .
\end{aligned}
$$

Приведем теперь интегралы Новикова $E_{k}$ :

$$
\begin{aligned}
& E_{1}=u^{(\mathrm{IV})}+5 u u^{\prime \prime}+\frac{15}{4} u^{\prime 2}+\frac{5}{3} u^{3}+c u, \\
& E_{2}=-\frac{1}{4} u^{\prime \prime \prime}\left(u^{\prime \prime \prime}+8 u u^{\prime}\right)-\frac{1}{4} u u^{\prime \prime 2}+\frac{1}{24} u^{\prime \prime}\left(E_{1}+3 u^{\prime 2}-20 u^{3}-12 c u\right)+\cdots, \\
& E_{3}=-\frac{3}{4} u^{\prime \prime \prime} u^{\prime}\left(u^{\prime \prime}+2 u^{2}+2 c\right)+\frac{1}{4} u^{\prime \prime 3}+\frac{3}{4}\left(u^{2}+c\right) u^{\prime \prime 2}-3 u^{\prime 2} u^{\prime \prime}+\cdots,
\end{aligned}
$$

где мы написали сокращенные выражения для $E_{2}, E_{3}$, так как они нам не понадобятся.

Переменные разделения $\gamma_{k}$ определяют полюсы логарифмической производной (8), т.е. факторизуют полином П. Поэтому мы полагаем по определению

$$
\Pi=3^{7}\left(\lambda-\gamma_{1}\right)\left(\lambda-\gamma_{2}\right)\left(\lambda-\gamma_{3}\right)\left(\lambda-\gamma_{4}\right)
$$

Если мы определим вторую координату $\mu_{k}=\mu_{k}(x)$ формулой

$$
\mu_{k}^{3}+Q\left(\gamma_{k}(x)\right) \mu_{k}+T\left(\gamma_{k}(x)\right)=0
$$

то из (8) получим аналог уравнений Дубровина для рассматриваемого случая. В самом деле, переходя к пределу $\lambda \rightarrow \gamma_{k}$ в (8), получаем

$$
\frac{d \gamma_{k}}{d x}=-\frac{\gamma_{k}}{81} \frac{3 \mu_{k}^{2}+Q\left(\gamma_{k}\right)}{\prod_{j \neq k}\left(\gamma_{k}-\gamma_{j}\right)}
$$


Дополнительные объяснения приведены в работе [3] и там же можно найти общий способ получения таких уравнений и второй координаты нуля полинома П:

$$
\mu_{k}=3 u \gamma_{k}+\frac{\left(u^{\prime \prime}+2 u^{2}+2 c\right)^{2}}{36 \gamma_{k}} .
$$

Сопоставляя (9) и (11), будем иметь, например, следующие тождества:

$$
u^{\prime}=6 \sum_{k=1}^{4} \gamma_{k}, \quad u^{(\mathrm{IV})}+5 u u^{\prime \prime}+4 u^{\prime 2}+2 u^{3}+2 c u=18 \sum_{k>j} \gamma_{k} \gamma_{j}
$$

Составляя симметрические комбинации уравнений (12) и используя формулу (13) для второй координаты, мы можем исключить все производные потенциала и получить линейное соотношение для $u$. Это и будет искомая рациональная формула следов. Опуская детали, имеем в результате

$$
u=\frac{1}{3} \sum_{k=1}^{4} \frac{\gamma_{k}^{2} \mu_{k}}{\prod_{j \neq k}\left(\gamma_{k}-\gamma_{j}\right)} .
$$

Последнее упрощение уравнений (12) - это сведение их к квадратурам. Вновь составляя симметрические комбинации правых частей (12), получаем уравнения

$$
\begin{array}{ll}
\sum_{k=1}^{4} \frac{d \gamma_{k}}{3 \mu_{k}^{2}+Q\left(\gamma_{k}\right)}=0, \quad \sum_{k=1}^{4} \frac{\gamma_{k} d \gamma_{k}}{3 \mu_{k}^{2}+Q\left(\gamma_{k}\right)}=0 \\
\sum_{k=1}^{4} \frac{\mu_{k} d \gamma_{k}}{3 \mu_{k}^{2}+Q\left(\gamma_{k}\right)}=0, \quad \sum_{k=1}^{4} \frac{\gamma_{k}^{2} d \gamma_{k}}{3 \mu_{k}^{2}+Q\left(\gamma_{k}\right)}=-\frac{1}{81} d x,
\end{array}
$$

которые, как и следовало ожидать, приводят к задаче Якоби, т.е. к задаче обращения четырех голоморфных интегралов на кривой (7):

$$
\begin{array}{rlrl}
\sum_{k=1}^{4} \int^{\left(\gamma_{k}, \mu_{k}\right)} \frac{d \lambda}{3 \mu^{2}+Q(\lambda)} & =a_{1}, & \sum_{k=1}^{4} \int^{\left(\gamma_{k}, \mu_{k}\right)} \frac{\mu d \lambda}{3 \mu^{2}+Q(\lambda)}=a_{3}, \\
\sum_{k=1}^{4} \int^{\left(\gamma_{k}, \mu_{k}\right)} \frac{\lambda d \lambda}{3 \mu^{2}+Q(\lambda)}=a_{2}, & \sum_{k=1}^{4} \int^{\left(\gamma_{k}, \mu_{k}\right)} \frac{\lambda^{2} d \lambda}{3 \mu^{2}+Q(\lambda)}=a_{4}-\frac{1}{81} x .
\end{array}
$$

Далее мы будем считать, что зависимость $\gamma_{k}=\gamma_{k}(x)$ определяется из этих уравнений.

Здесь стоит добавить, что подобная схема возникновения уравнений Дубровина и формул следов из определения дифференциального полинома П и функции $\Psi$ является характерной для общих конечнозонных операторов. Определения величин $\gamma_{k}$, уравнения Дубровина, всевозможные соотношения для потенциала и его производных (разные версии формул следов) и т.д. “одновременно перепутаны” в общем наборе определяющих полиномиальных соотношений. 


\section{3. ТРАНСЦЕНДЕНТНАЯ ФОРМУЛА СЛЕДОВ}

Вывод предыдущих формул, как мы видим, содержит манипуляции полиномами, что характерно для теории полиномиальных идеалов [6]. Базисы таких идеалов не единственны, и можно ожидать, что найдутся другие соотношения, которые определяют потенциал. Это действительно так. Исключим из интеграла Новикова $E_{1}$ и второго тождества в (14) старшую производную $u^{(\mathrm{IV})}$. Получим квадратное уравнение на $u^{\prime}$ :

$$
3 u^{\prime 2}+4 u^{3}+12 c u+E_{1}=216 \sum_{k>j} \gamma_{k} \gamma_{j} .
$$

Но $u^{\prime}$ выражается однозначно через $\gamma$ в силу первого тождества в (14). Тогда мы получаем кубический полином по переменной $u$ :

$$
u^{3}+3 c u+3 E_{1}+27 \sum_{k=1}^{4} \gamma_{k}^{2}=0
$$

который можно воспринимать как алгебраический вариант главной формулы следов.

С другой стороны, конечнозонные потенциалы суть однозначные функции переменной $x$. Они удовлетворяют автономным обыкновенным дифференциальным уравнениям, проходят тест Пенлеве и выражаются через тета-функции. Известно также (но редко используется), что корни всякого полинома третьей или четвертой степени могут быть представлены в виде аналитических выражений от своих коэффициентов в терминах эллиптических функций [7]. В случае полинома (16) ситуация проще, так как мы сразу можем рассматривать его как полином Вейерштрасса:

$$
4 u^{3}+12 c u+12 E_{1}+108 \sum_{k=1}^{4} \gamma_{k}^{2}(x)=4 u^{3}-a u-b=4(u-e)\left(u-e^{\prime}\right)\left(u-e^{\prime \prime}\right),
$$

где точки $e, e^{\prime}, e^{\prime \prime}$ зависят от коэффициентов $a, b$, а коэффициенты - от $x$. Корни этого полинома $u=\wp\left(\omega_{k}(x) ; a, b\right)$, где

$$
a=-12 c, \quad b=-108 \sum \gamma_{k}^{2}(x)-12 E_{1},
$$

выражаются через тета-константы [7]:

$$
\begin{aligned}
e & =\frac{1}{\omega^{2}} \frac{\pi^{2}}{12}\left\{\vartheta_{3}^{4}(\tau)+\vartheta_{4}^{4}(\tau)\right\}, \\
e^{\prime} & =-\frac{1}{\omega^{2}} \frac{\pi^{2}}{12}\left\{\vartheta_{2}^{4}(\tau)+\vartheta_{3}^{4}(\tau)\right\}, \\
e^{\prime \prime} & =\frac{1}{\omega^{2}} \frac{\pi^{2}}{12}\left\{\vartheta_{2}^{4}(\tau)-\vartheta_{4}^{4}(\tau)\right\},
\end{aligned}
$$

а величины $\omega$ и $\tau=\omega^{\prime} / \omega$ следует выразить через коэффициенты $a$ и $b$. Достаточно взять одно из таких выражений, а присутствующие в (17) якобиевские $\vartheta$-константы 
записать с помощью классических рядов [7]:

$$
\begin{aligned}
& \vartheta_{2}(\tau)=\mathrm{e}^{\pi i \tau / 4} \sum_{k=-\infty}^{\infty} \mathrm{e}^{\left(k^{2}+k\right) \pi i \tau}, \\
& \vartheta_{3}(\tau)=\sum_{k=-\infty}^{\infty} \mathrm{e}^{k^{2} \pi i \tau}, \\
& \vartheta_{4}(\tau)=\sum_{k=-\infty}^{\infty}(-1)^{k} \mathrm{e}^{k^{2} \pi i \tau}
\end{aligned}
$$

(мы используем символ “е” для экспоненты, чтобы отличать ее от точек Вейерштрасса).

Итак, мы пришли к классической эллиптической модулярной задаче обращения, т.е. к задаче нахождения периодов $\left(2 \omega, 2 \omega^{\prime}\right)$ эллиптической кривой $w^{2}=4 z^{3}-a z-b$ по ее коэффициентам. Схема решения известна. Необходимо найти корень $\tau$ трансцендентного уравнения $J(\tau)=a^{3} /\left(a^{3}-27 b^{2}\right)$, где $J(\tau)$ - классическая модулярная функция Клейна [7], [8]. После того как корень $\tau$ найден, полупериоды $\left(\omega, \omega^{\prime}\right)$ вычисляются по формулам

$$
\omega^{2}=\frac{a}{b} \frac{g_{3}(\tau)}{g_{2}(\tau)}, \quad \omega^{\prime}=\tau \omega,
$$

где $g_{2,3}(\tau)$ - известные модулярные формы [8]. Для них имеются ряды Эйзенштейна, ряды Гурвица-Ламберта или представления через $\vartheta$-константы:

$$
\begin{aligned}
g_{2}(\tau) & =\frac{\pi^{4}}{24}\left\{\vartheta_{2}^{8}(\tau)+\vartheta_{3}^{8}(\tau)+\vartheta_{4}^{8}(\tau)\right\} \\
g_{3}(\tau) & =\frac{\pi^{6}}{432}\left\{\vartheta_{2}^{4}(\tau)+\vartheta_{3}^{4}(\tau)\right\}\left\{\vartheta_{3}^{4}(\tau)+\vartheta_{4}^{4}(\tau)\right\}\left\{\vartheta_{4}^{4}(\tau)-\vartheta_{2}^{4}(\tau)\right\}
\end{aligned}
$$

Осталось выписать выражение для корня $\tau$. Как ни странно, но явное аналитическое решение столь классической задачи отсутствует ${ }^{1)}$, хотя общеизвестно, что его можно записать через отношения гипергеометрических ${ }_{2} F_{1}$-рядов. В силу того что ряд ${ }_{2} F_{1}(J)$ сходится только в единичном круге, формулы будут различны в зависимости от того, какому из неравенств $|J|>1$ или $|J|<1$ удовлетворяет $J$. Всё вместе, это составляет совокупность довольно громоздких выражений, включающих к тому же функциональные ряды по логарифмической производной от Г-функции Эйлера. Например, соответствующие формулы (22)-(27) в п. 14.6.2 книги [8] занимают полстраницы (не считая некорректности в формуле (23)). Оперировать ими аналитически не представляется возможным. Между тем задача имеет простое решение.

Воспользуемся хорошо известным фактом, что функция $J$ тесно связана с гипергеометрическим уравнением вида

$$
J(J-1) \psi^{\prime \prime}+\frac{1}{6}(7 J-4) \psi^{\prime}+\frac{1}{144} \psi=0 .
$$

\footnotetext{
1) K сожалению, в энциклопедии [9] на с. 789 и в замечательной во всех отношениях книге [7] на с. 51 решения этой задачи изложены неверно, и это не связано с опечатками.
} 
Решения гипергеометрических уравнений в общем случае являются гипергеометрическими функциями ${ }_{2} F_{1}(a, b ; c \mid z)$, но при определенных условиях на параметры $a, b, c$ они представимы через классические специальные функции. Мы имеем в виду не вырождения функции ${ }_{2} F_{1}$, а случаи, когда ${ }_{2} F_{1}$-ряд допускает квадратичные преобразования и поэтому гипергеометрическое уравнение сводится к уравнению с двумя параметрами, т.е. к уравнению Лежандра. Именно это справедливо для выписанного выше уравнения. Его решение есть линейная комбинация

$$
\psi=\sqrt[6]{J}\left\{A P_{\nu}^{\mu}(\sqrt{1-J})+B Q_{\nu}^{\mu}(\sqrt{1-J})\right\}
$$

функций Лежандра с параметрами $\nu=-1 / 2$ и $\mu=1 / 3$. Исчерпывающую информацию об этих функциях см. в книге [10]. Сказанное выше означает, что должна иметь место формула

$$
\tau=\frac{\mathbf{a} P(\sqrt{1-J})+\mathbf{b} Q(\sqrt{1-J})}{\mathbf{c} P(\sqrt{1-J})+\mathbf{d} Q(\sqrt{1-J})}
$$

с определенными числовыми значениями для $\mathbf{a}, \mathbf{b}, \mathbf{c}, \mathbf{d}$ (индексы $\nu, \mu$ у функций Лежандра мы для краткости опускаем). Приведем окончательный ответ, а его вывод будет изложен в другой работе.

ПрЕДЛОЖЕНИЕ. Для эллиптической кривой $w^{2}=4 z^{3}-a z-b$ отношение периодов $\tau=\omega^{\prime} / \omega$ дается следующим выражением:

$$
\tau=\left\{\pi i \frac{P(\sqrt{1-\mathbf{J}})}{Q(\sqrt{1-\mathbf{J}})}-1\right\} \mathrm{e}^{\pi i / 3}, \quad \mathbf{J}=\frac{a^{3}}{a^{3}-27 b^{2}} .
$$

ЗАмЕчаниЕ. Если в равенство (21) вместо Ј подставить $J(\tau)$, то это равенство превратится в тождество для любых $\tau$ из верхней полуплоскости $\mathbb{H}^{+}$. Левую и правую части этого тождества следует, конечно, вычислять по модулю полной модулярной группы $P S L_{2}(\mathbb{Z})=: \boldsymbol{\Gamma}(1)$. Таким образом, соотношение $(21)$ является $\boldsymbol{\Gamma}(1)$-эквивалентом решения модулярной задачи обращения в представлении Лежандра. А именно, если эллиптическая кривая задана в форме Лежандра $w^{2}=\left(1-z^{2}\right)\left(1-k^{2} z^{2}\right)$, то эллиптический модуль $\tau$ вычисляется по знаменитой формуле Якоби

$$
\tau=i \frac{K^{\prime}(k)}{K(k)} \bmod \boldsymbol{\Gamma}(2)
$$

где $K$ и $K^{\prime}$ - полные эллиптические интегралы Лежандра первого рода [7], [8], [11].

Теперь, возвращаясь к потенциалу, по формулам (17), (18) с учетом (19) получаем искомую трансцендентную “формулу следов":

$$
u(x)=\frac{3}{2 c}\left\{E_{1}+9 \sum_{k=1}^{4} \gamma_{k}^{2}(x)\right\} \frac{\vartheta_{2}^{8}(\tau)+\vartheta_{3}^{8}(\tau)+\vartheta_{4}^{8}(\tau)}{\left\{\vartheta_{2}^{4}(\tau)+\vartheta_{3}^{4}(\tau)\right\}\left\{\vartheta_{4}^{4}(\tau)-\vartheta_{2}^{4}(\tau)\right\}}
$$


где под $\tau$ следует понимать функцию

$$
\tau(x)=\widehat{\boldsymbol{\Gamma}}\left(\pi i \frac{P(\sqrt{1-\mathbf{J}})}{Q(\sqrt{1-\mathbf{J}})} \mathrm{e}^{\pi i / 3}-\mathrm{e}^{\pi i / 3}\right),
$$

в которой

$$
\mathbf{J}=\frac{4 c^{3}}{4 c^{3}+9\left\{E_{1}+9 \sum \gamma_{k}^{2}(x)\right\}^{2}},
$$

а символом $\widehat{\boldsymbol{\Gamma}}$ мы обозначили операцию приведения числа в фундаментальную область группы. Формулы $(23),(24)$ не содержат координаты $\mu_{k}$. Хорошим и очень нетривиальным упражнением является прямая проверка того, что функция (23) действительно удовлетворяет соотношению (10) или стационарной версии уравнения (4).

\section{4. ТРАНСЦЕНДЕНТНЫЕ ТОЖДЕСТВА МЕЖДУ ТЕТА-ФУНКЦИЯМИ}

Как следует из предыдущего раздела, аналогичным способом можно получить много других примеров абелевых функций, являющихся радикалами или корнями алгебраических уравнений. Например, из последнего слагаемого в полиноме (9) следует однозначное представление радикала абелевой функции вида $18 \sqrt[3]{\gamma_{1} \gamma_{2} \gamma_{3} \gamma_{4}}=$ $u^{\prime \prime}+2 u^{2}+2 c$, а общий механизм получения “трансцендентных следов" состоит в исключении производных от потенциала из полного набора соотношений, определяющих интегралы $E_{k}$ и переменные $\gamma_{k}$. Разрешая получающиеся алгебраические уравнения в однозначных функциях (а это всегда тета-функции), получаем ответ. Эти уравнения (возможно, даже и их порядки) не единственны и зависят от рода, но переходы между ними есть не что иное, как различные представления одной и той же абелевой функции. Даже в $(\gamma, \mu)$-представлении они различны, так как координаты $\gamma$ и $\mu$ связаны алгебраическим соотношением.

Если мы теперь приравняем трансцендентное и рациональное представления, то получим необычное тождество, содержащее тета-функции. В самом деле, сопоставив, например, (15) и (23),

$$
\frac{1}{3} \sum_{k=1}^{4} \frac{\gamma_{k}^{2} \mu_{k}}{\prod_{j \neq k}\left(\gamma_{k}-\gamma_{j}\right)}=\frac{3}{2 c}\left\{E_{1}+9 \sum_{k=1}^{4} \gamma_{k}^{2}\right\} \frac{\vartheta_{2}^{8}(\tau)+\vartheta_{3}^{8}(\tau)+\vartheta_{4}^{8}(\tau)}{\left\{\vartheta_{2}^{4}(\tau)+\vartheta_{3}^{4}(\tau)\right\}\left\{\vartheta_{4}^{4}(\tau)-\vartheta_{2}^{4}(\tau)\right\}}
$$

мы можем, в принципе, придать входящим в это равенство рациональным по $(\gamma, \mu)$ абелевым функциям форму $\Theta$-функциональных отношений [4]. Получившееся соотношение между $\Theta$-функциями рода $g=4$ будет трансцендентным тождеством, включающим эллиптические тета-константы $\vartheta(\tau(x))$, хотя кривая $(7)$, вообще говоря, не имеет отношения ни к какой эллиптической кривой. Помимо модулей кривой, тождество (25) содержит произвольный параметр $x$. Можно также перенести в левую часть равенства все симметрические рациональные по $(\gamma, \mu)$ функции. Тогда слева мы получим некоторую абелеву функцию на якобиане тригональной кривой (7), а справа - ее “экзотическое” представление через эллиптические 
тета-константы от функций Лежандра:

$$
u^{2}=-2 c \frac{\left\{\vartheta_{2}^{4}(\tau)+\vartheta_{3}^{4}(\tau)\right\}\left\{\vartheta_{4}^{4}(\tau)-\vartheta_{2}^{4}(\tau)\right\}}{\vartheta_{2}^{8}(\tau)+\vartheta_{3}^{8}(\tau)+\vartheta_{4}^{8}(\tau)}-3 c .
$$

В свою очередь, аргументами входящих в это представление функций Лежандра (гипергеометрических функций) будут выражения, содержащие по формуле (24) через величину $\sqrt{1-\mathbf{J}}$ другую абелеву функцию, т.е. $\sum \gamma_{k}^{2}(x)$. Она представляется своей рациональной дробью от $\Theta$-функций типа $\Theta(x \mathbf{U}+\mathbf{D})$. Характер трансцендентных соотношений, как видим, вообще говоря, очень сложный и его еще следует понять. Даже если якобиан кривой (7) полностью расщепляется на эллиптические кривые, и многомерные $\Theta$-функции превращаются в эллиптические $\theta$-функции, трансцендентность сохраняется. Полиномы типа (16) при этом не вырождаются, и поэтому естественно ожидать появления трансцендентных $\theta$-тождеств.

Тот вид трансцендентности, который получен выше, связан с эллиптическим модулярным обращением, поскольку оно происходит от решения алгебраического уравнения с использованием эллиптических модулярных функций. Но это не единственный способ решения таких уравнений. В более общих методах привлекаются общие тета-функции и константы (см., например, статью Умемуры в книге [12]), поэтому естественно ожидать появления других разновидностей трансцендентных тождеств.

В заключение отметим, что одновременное и трансцендентное сочетание тетафункций и гипергеометрических рядов на самом деле не так уж неожиданно, поскольку естественным атрибутом ${ }_{2} F_{1}$-рядов являются группы, известные как монодромии, а они, в свою очередь, представляют автоморфизмы аналитических автоморфных функций. Все такие функции, которые известны в настоящее время, выглядят как отношения тета-констант. Выражаясь неформально, "тета" и "гипергеометрия" взаимно обратны друг к другу (они реализуют обращения отношений типа (20)), а наши трансцендентные тождества отражают как этот факт, так и свойства функций типа $\Theta(x \mathbf{U}+\mathbf{D})$. А именно, абелевы многообразия, как алгебраические многообразия, параметризуются полиномами по тета-функциям, а линейные сечения якобианов возникают из интегрируемых уравнений. Частичную иллюстрацию дает, например, уже якобиевское уравнение (22), если его переписать в виде

$$
\tau \equiv i \frac{K^{\prime}\left(\vartheta_{2}^{2}(\tau) / \vartheta_{3}^{2}(\tau)\right)}{K\left(\vartheta_{2}^{2}(\tau) / \vartheta_{3}^{2}(\tau)\right)} \quad \bmod \quad \boldsymbol{\Gamma}(2)
$$

для любого $\tau \in \mathbb{H}^{+}$. Рассматривая это уравнение в достаточно малой окрестности некоторой точки $\tau_{0}$, "не вполне аналитическую" операцию приведения точки в фундаментальную область группы можно отбросить, и оно превратится в точное аналитическое трансцендентное тождество. Аналогично можно поступить и с тета-версиями наших соотношений $(25),(26)$ как с тождествами по $x$.

Благодарности. Работа поддержана Федеральным агентством по науке и инновациям РФ (грант № 02.740.11.0238) и Программой поддержки ведущих научных школ (грант НШ-3400.2010.2). 


\section{Список литературы}

[1] L. A. Dickey, Solitons Equations and Hamiltonian Systems, Adv. Ser. Math. Phys., 26, World Sci., Singapore, 2003.

[2] M. Błaszak, Multi-Hamiltonian Theory of Dynamical Systems, Texts Monogr. Phys., Springer, Berlin, 1998.

[3] Ю. В. Брежнев, УМН, 57:2(344) (2002), 191-192.

[4] В. М. Бухштабер, Д. В. Лейкин, В. З. Энольский, Функи. анализ и его прил., 34:3 (2000), 1-16.

[5] D. J. Kaup, Stud. Appl. Math., 62:3 (1980), 189-216.

[6] Д. Кокс, Дж. Литтл, Д. О’Ши, Идеаль, многообразия и алгоритмы, Мир, М., 2000.

[7] Н. И. Ахиезер, Элементы теории эллиптических функций, Наука, М., 1970.

[8] Г. Бейтмен, А. Эрдейи, Высшие трансиендентные функиии. Эллиптические и автоморфные функиии. Функиии Ламе и Матъе, Справочная математическая библиотека, Наука, М., 1967.

[9] И. М. Виноградов (ред.), Математическая энииклопедия, т. III, Советская энциклопедия, М., 1982.

[10] Г. Бейтмен, А. Эрдейи, Высшие трансиендентные функиии, т. I, Гипергеометрическая функция. Функции Лежандра, Наука, М., 1973.

[11] Э. Т. Уиттекер, Дж. Н. Ватсон, Курс современного анализа, ч. 2, Физматлит, М., 1963.

[12] Д. Мамфорд, Лекиии о тэта-функииях, Мир, М., 1988.

Поступила в редакцию 16.12.2009 